\title{
Thermal Analysis of Natural Convection in Porous Fins with Homotopy Perturbation Method (HPM)
}

\author{
Seyfolah Saedodin · Majid Shahbabaei
}

Received: 27 August 2011 / Accepted: 23 September 2011 / Published online: 20 February 2013

(C) The Author(s) 2013. This article is published with open access at Springerlink.com

\begin{abstract}
In this paper, porous fin has been studied and its nonlinear ordinary differential equation has been solved through homotopy perturbation method. In this method, a homotopy is introduced to be constructed for the equation. The initial approximations can be freely chosen with possible unknown constants, which can be determined by imposing the boundary and initial conditions. To study the thermal performance, one type case is considered: finite-length fin with insulated tip.
\end{abstract}

Keywords Porous fin - Homotopy perturbation method $(\mathrm{HPM}) \cdot$ Nonlinear ordinary differential equation . Finite-length fin with insulated tip

S. Saedodin

Faculty of Mechanical Engineering, Semnan University,

Semnan, Iran

e-mail: S_saedodin@iust.ac.ir

M. Shahbabaei ( $\varangle)$

Faculty of Mechanical Engineering, Science and Researches,

Semnan Branch, Semnan, Iran

e-mail: Majid0123@gmail.com

\author{
Nomenclature \\ k Thermal conductivity \\ Da Darcy number, K/t2 \\ $\mathrm{Kr} \quad$ Thermal conductivity ratio, $\left(k_{e f f} / k_{f}\right)$ \\ $\mathrm{K}$ Permeability of porous fin \\ L Length \\ m convection parameter \\ q Heat transfer rate $\alpha$ \\ Ra Rayleigh number, $\mathrm{Gr} \times \operatorname{Pr}$ \\ Sh Porous parameter \\ $\mathrm{T}(\mathrm{x}) \quad$ Temperature at any point \\ $\mathrm{Tb}$ Temperature at fin base \\ $\mathrm{t} \quad$ Thickness of the fin \\ $v_{W(x)}$ Velocity of fluid passing through the fin anypoint \\ W Width of the fin \\ X Axial coordinate \\ $\mathrm{X}$ Dimensionless axial coordinate, $(x / L)$ \\ $\theta \quad$ Dimensionless temperature, \\ $\theta b \quad$ Base temperature difference, $(\mathrm{Tb}-\mathrm{T} \infty)$ \\ s Solid properties \\ f $\quad$ Fluid properties \\ eff Porous properties
}

\section{Introduction}

The homotopy perturbation method (HPM) was first proposed by He [14-24]. The HPM does not depend on a small parameter in the equation. Using homotopy technique in topology, a homotopy is constructed with an embedding parameter $p \in[0,1]$, which is considered as a "small parameter". The HPM was successfully applied to nonlinear oscillators with discontinuities [1-4] and bifurcation of nonlinear problems [5]. In [2], a comparison of HPM and homotopy analysis method was made. Most of the scientific 
problems and phenomena occur nonlinearly. Except a limited number of these problems, most of them do not have precise analytical solutions so that they have to be solved using other methods. Many different new methods have recently presented some techniques to eliminate the small parameter; for example, the variational iteration method (VIM) [6-9] and the Exact solutions (ADM) [10,11], HPM [12-17], and others $[18,19]$. In [25], a new perturbation algorithm combining the method of multiple scales and lindstedt-Poincare techniques is proposed for first time. The new perturbation algorithm combining the method of multiple scales (MS) and lindstedt-Poincare techniques is applied to an equation with quadratic and cubic nonlinearities [26] . In [27], forced vibrations of duffing equation with damping is considered. Paper [28] shows two new perturbation-iteration algorithms for solving differential equations of first order. A nonlinear fin equation in which thermal conductivity is an arbitrary function of temperature and heat transfer coefficient is an arbitrary function of spatial variable is considered [29], [30]. Scaling, translational and spiral group symmetries of the equations are determined. In [31], they propose an analytical solution to a nonlinear second-order boundary value problem using HPM and $4 \mathrm{q}-$ Boubaker polynomials Expansion scheme (BPES). In [32], they investigated magnetohydrodynamic (MHD) viscous flow due to a shrinking sheet by employing the HPM and pade approximation. A series solution of the long porous slider problem [33] where fluid is injected through the porous bottom is obtained using the HPM. In this article, HPM is used to solve nonlinear equations of finite-length fin with insulated tip with the initial conditions $\theta(0)=1, \theta^{\prime}(1)=0$ for the first time.

\section{Material and Methods}

As shown in Fig. 1, a rectangular fin profile is considered. The dimensions of the fin are length $L$, width $w$ and thickness $t$. The cross section area of the fin is constant. This fin is porous to allow the flow of infiltrate through it. The following assumptions are made to solve this problem.

From the Darcy's model, we have:

$v_{w}=\frac{g k \beta\left(T-T_{\infty}\right)}{v}$

Now applying energy balance equation at steady state condition, we have:

$\frac{d^{2} \theta}{d x^{2}}-S_{h} \theta(x)^{2}-m^{2} \theta(x)=0$

Where, $\theta=\frac{T(x)-T_{\infty}}{T_{b}-T_{\infty}}$ and $X=\frac{x}{L}$

Porous parameter, $S_{h}=\frac{\operatorname{Dax} R a}{k_{r}}\left(\frac{L}{t}\right)^{2}$ and Convection parameter, $m=\left(\frac{h p}{k_{s} A}\right)^{\frac{1}{2}}$

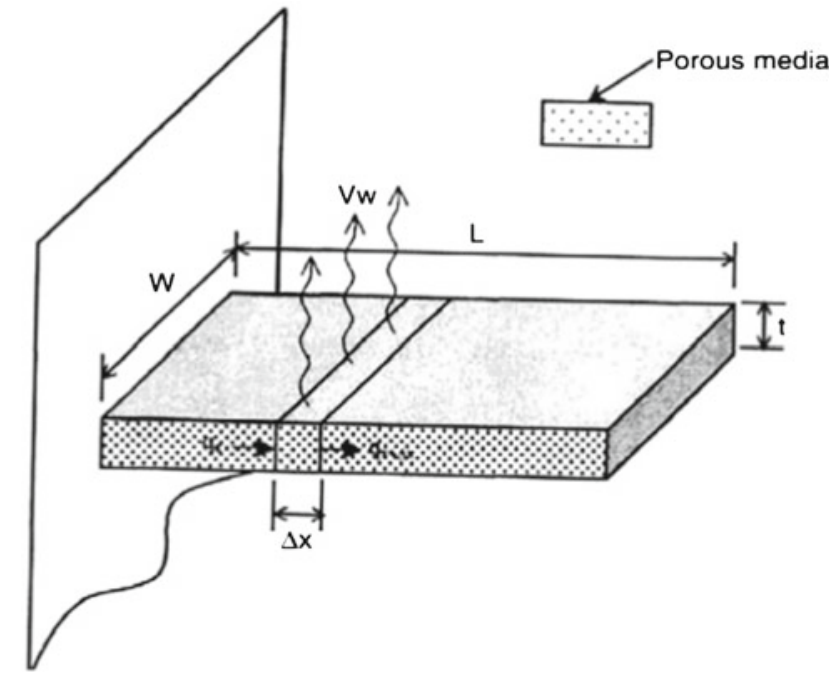

Fig. 1 Schematic diagram of fin profile under consideration

Here, $s_{h}$ is a porous parameter that indicates the effect of the permeability of the porous medium as well as buoyancy effect, so higher value of $s_{h}$ indicates higher permeability of the porous medium or higher buoyancy forces. $\mathrm{m}$ is a convection parameter that indicates the effect of surface convecting of the fin.

Here is the summary of case to be considered for this research.

Case: finite-length fin with insulated tip $(\theta(0)=1$, $\mathrm{d} \theta /\left.\mathrm{dx}\right|_{x=1}=0$ )

\section{Basic Concept of He's Homotopy Perturbation Method}

To illustrate the basic ideas of this method, we consider the following equation:

$\mathrm{A}(\mathrm{F})-\mathrm{f}(\mathrm{r})=0 \quad \mathrm{r} \in \Omega$

With the boundary condition of:

$\mathrm{B}\left(\mathrm{u}, \frac{\partial \mathrm{F}}{\partial \mathrm{n}}\right)=0$

Where $A$ is a general differential operator, $B$ a boundary operator, $f(r)$ a known analytical function and $\Gamma$ is the boundary of the domain $\Omega$. A can be divided into two parts, $L$ and $N$, where $L$ is linear and $N$ is nonlinear. Equation 2 can, therefore, be rewritten as follows:

$\mathrm{L}(\mathrm{F})+\mathrm{N}(\mathrm{F})-\mathrm{f}(\mathrm{r})=0 \quad \mathrm{r} \in \Omega$

Homotopy perturbation structure is shown as follows:

$\mathrm{H}(V, P)=(1-P)\left[L(V)-L\left(u_{0}\right)\right]+P[A(V)-f(r)]=0$ 
Where,

$V(r, p): \Omega \times[0,1] \rightarrow R$,

In Eq. (6), $p \in[0,1]$ is an embedding parameter and $u_{0}$ is the first approximation that satisfies the boundary condition. We can assume that the solution of Eq. (1) can be written as a power series in $p$, as following:

$V=V_{0}+P V_{1}+P^{2} V_{2}+\cdots=\sum_{i=0}^{n} V_{i} P^{i}$

And the best approximation for solution is:

$F=\lim _{P \rightarrow 1} V=V_{0}+V_{1}+V_{2}+\cdots$

\section{Application of HPM to Porous Fin}

In this section, we will apply the HPM to nonlinear ordinary differential Eq. (1). According to the HPM, we can construct homotopy of Eq. (1) as follows:

$$
\begin{aligned}
&(1-p), {\left[\frac{d^{2}}{d x^{2}} \theta(x)-\theta(x)\right] } \\
&+p \cdot\left[\frac{d^{2}}{d x^{2}} \theta(x)-\theta(x)^{2}-\theta(x)\right]=0
\end{aligned}
$$

We consider $\theta$ as follows:

$\theta(x)=\theta_{0}(x)+\theta_{1}(x)+\theta_{2}(x)+\cdots=\sum_{i=0}^{n} \theta_{i}(x)$

Substituting $\theta$ from Eq. (10) into Eq. (9) and some simplification and rearranging based on powers of $p$-terms, we have:

$$
\begin{aligned}
& P^{0}:-\theta_{0}(x)+\frac{d^{2}}{d x^{2}} \theta_{0}(x)=0, \quad \theta_{0}(0)=1, \quad \theta_{0}^{\prime}(1)=0 \\
& P^{1}:-\theta_{0}(X)^{2}-\theta_{1}(X)+\frac{d^{2}}{d x} \theta_{1}(X)=0, \theta_{1}(0)=0, \theta_{1}^{\prime}(1)=0 \\
& P^{2}:-2 \theta_{0}(X) \theta_{1}(X)=0, \theta_{2}(0)=0, \theta_{2}^{\prime}(1)=0 \\
& P^{3}:-\theta_{1}(X)^{2}=0, \theta_{3}(0)=0, \theta_{3}^{\prime}(1)=0
\end{aligned}
$$

Solving Eqs. (11)-(14) with boundary conditions, we have:

$$
\begin{aligned}
\theta_{a}(\mathrm{X})= & \frac{\mathrm{e} \mathrm{e}^{-\mathrm{X}}}{\mathrm{e}^{-1}+\mathrm{e}}+\frac{\mathrm{e}^{-1} \mathrm{e}^{\mathrm{X}}}{\mathrm{e}^{-1}+\mathrm{e}} \\
\theta_{1}(\mathrm{X})= & -\frac{1}{3} \frac{\mathrm{e}^{-\mathrm{X}} \mathrm{e}\left(\mathrm{e}^{2}-6+\mathrm{e}^{-2}\right) \mathrm{e}^{2}}{\mathrm{e}^{4} \mathrm{e}^{-1}+2 \mathrm{e}^{2} \mathrm{e}^{-1}+\mathrm{e}^{-1}+\mathrm{e}+2 \mathrm{ee}^{2}+\mathrm{ee}^{4}} \\
& -\frac{1}{3} \frac{\mathrm{e}^{\mathrm{X}} \mathrm{e}^{-1}\left(\mathrm{e}^{2}-6+\mathrm{e}^{-2}\right) \mathrm{e}^{2}}{\mathrm{e}^{-1}+2 \mathrm{e}^{2} \mathrm{e}^{-1}+\mathrm{e}^{-1}+\mathrm{e}+2 \mathrm{ee}^{2}+\mathrm{ee}^{4}} \\
& +\frac{\left(\mathrm{e}^{2}-6 \mathrm{e}^{2 \mathrm{X}}+\mathrm{e}^{4 \mathrm{X}-2}\right) \mathrm{e}^{-2 \mathrm{X}+2}}{3+6 \mathrm{e}^{2}+3 \mathrm{e}^{4}}
\end{aligned}
$$

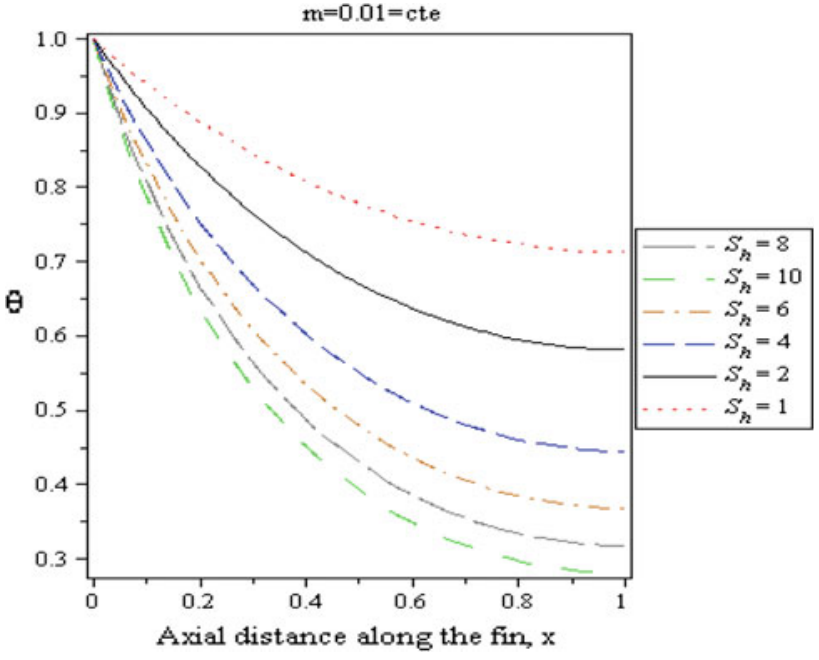

Fig. 2 The distribution of axial non-dimensional temperature along the finite-length fin for different values of $\mathrm{S}_{h}$

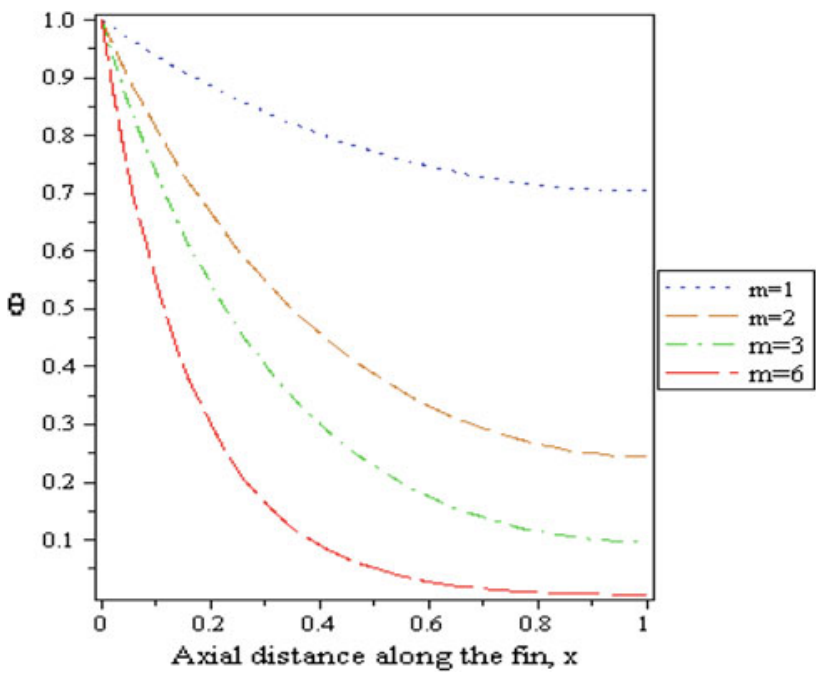

Fig. 3 The distribution of axial non-dimensional temperature along the finite-length fin for different values of $m$

The solution of this equation, when $p \rightarrow 1$, will be as follows:

$\theta(\mathbf{X})=\theta_{0}(\mathbf{X})+\theta_{1}(\mathbf{X})$

Hence, the equation answer to set boundary conditions will be:

$$
\left[-4 / 3 \frac{e^{6}+3 e^{4}-3 e^{2}-1}{\left(1+2 e^{2}+e^{4}\right)\left(1+e^{2}\right)}\right]
$$

\section{Results and Discussion}

The governing Eq. (1) is a non-linear second-order ordinary differential equation. The equation is solved numerically 
Fig. 4 The variation of the ratio of porous fin to solid fin heat transfer rate with $\mathrm{Kr}$

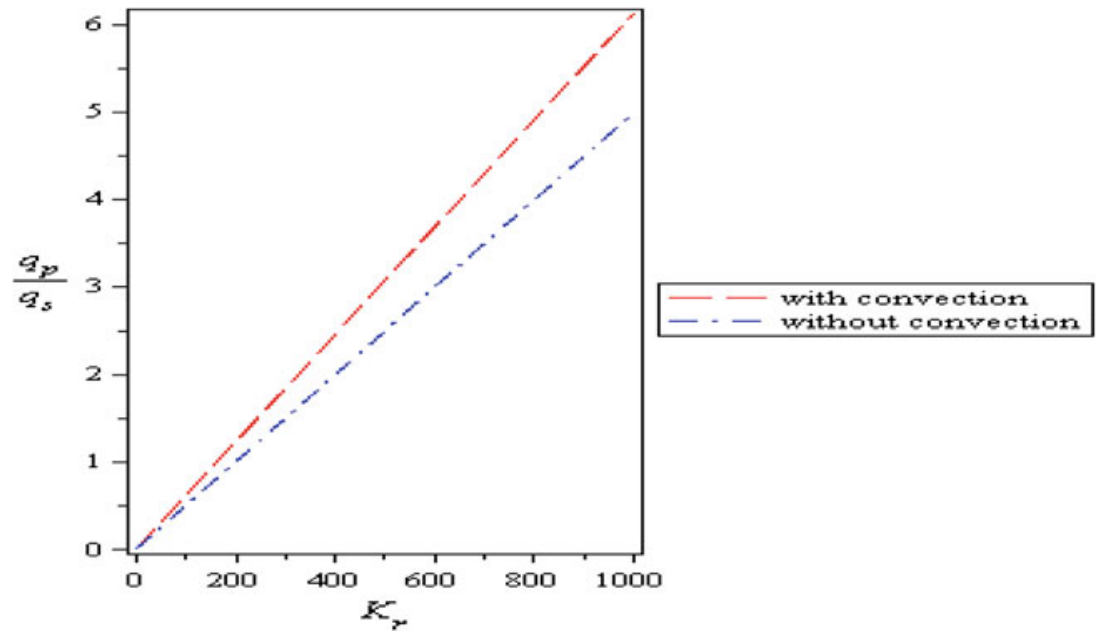

using the by HPM. Depending on the tip condition of the fin, we have three different types of cases, but in this research, we only study finite-length fin with insulated tip.

From Fig. 2, we can see that the value of dimensionless temperature decreases along the fin length. It should be noted that as the value of $S_{h}$ increases, the temperature decreases rapidly and the fin quickly reaches the surrounding temperature.

Figure 3 shows the results for the effect of variation of $m$ on dimensionless temperature distribution. The values of $m$ were varied while keeping $S_{h}=1$. Figure 4 shows porous fin to solid fin heat transfer rate with convection and without convection. The figure also shows the variation ratio of porous fin to solid fin heat transfer rate with $k_{r}$. Variation of ratio of porous fin to solid fin heat transfer rate increases as $k_{r}$ increases for both the cases.

\section{Conclusions}

This work introduces a simple method to analysis the performance of a porous fin. It is found that the problem of heat transfer through the porous fin is governed by a secondorder nonlinear, ordinary, differential equation. In this letter, we have studied porous fin problem with a small parameter with the homotopy perturbation method. As shown in Eq. (6), the homotopy perturbation method does not need a small parameter. This thermal analysis was performed on one type of fin case: The finite-length fin with insulated tip. The effect of these all two parameters $m$ and $S_{h}$ has been investigated.

Acknowledgments Authors are grateful to the all of people support them to carry out this work.
Open Access This article is distributed under the terms of the Creative Commons Attribution License which permits any use, distribution, and reproduction in any medium, provided the original author(s) and the source are credited.

\section{References}

1. Jeffery, G.B.: The two-dimensional steady motion of a viscous fluid. Phil. Mag. 6, 455-465 (1915)

2. Hamel, G.: Spiralförmige Bewgungen Zäher Flüssigkeiten. Jahresber Deutsch Math- Verein. 25, 34-60 (1916)

3. Rosenhead, L.: The steady two-dimensional radial flow of viscous fluid between two inclined plane walls. Proc. R. Soc. A. 175, 436467 (1940)

4. Batchelor, K.: An Introduction to Fluid Dynamics. Cambridge University Press, London (1967)

5. Sobey, I.J.; Drazin, P.G.: Bifurcations of two-dimensional channel flows. J. Fluid Mech. 171. 263-287 (1986)

6. Hamadiche, M.; Scott, J.; Jeandel, D.: Temporal stability of JefferyHamel flow. J. Fluid Mech. 268, 71-88 (1994)

7. Fraenkel, L.E.: Laminar flow in symmetrical channels with slightly curved walls.on the Jeffery-Hamel solutions for flow between plane walls. Proc. R. Soc. Lond. A. 267, 119-138 (1962)

8. Makinde, O.D.; Mhone, P.Y.: Hermite-Padé approximation approach to MHD Jeffery- Hamel flows. Appl. Math. Comput. 181, 966-972 (2006)

9. Schlichting, H.: Boundary-layer theory. McGraw-Hill Press, New York (2000)

10. Rathy, R.K.: An introduction to fluid dynamics. Oxford and IBH Pl, New Delhi (1976)

11. McAlpine, A.; Drazin, P.G.: On the spatio-temporal development of small perturbations of Jeffery-Hamel flows. Fluid Dynam. Res. 22, 123-138 (1998)

12. Tolou, N.; Ganji, D.D.; Hosseini, M.J.; Ganji, Z.Z.: Application of homotopy perturbation method in nonlinear heat diffusionconvection-reaction equations, Open Mech. J. 1, 20-25 (2007)

13. Jamshidi, N.; Ganji, D.D.; Ganji, Z.Z.: HPM and VIM methods for finding the exact solutions of the nonlinear dispersive equations and seventh-order Sawada-Kotara equation, Int. J. Modern Phys. B 23(1), 39-52 (2009)

14. He, J.H.: Homotopy perturbation method: a new nonlinear analytical technique, J. Appl. Math. Comput. 135, 73-79 (2003) 
15. He, J.H.: The Homotopy perturbation method for nonlinear oscillators with discontinuities. J. Appl. Math. Comput. 151, 287-292 (2004)

16. Ganji, D.D.; Sadighi, A.: Application of homotopy-perturbation and variational iteration methods to nonlinear heat transfer and porous media equations, J. Comput. Appl. Math. 207, 24-34 (2007)

17. Ganji, D.D.: The application of Hes homotopy-perturbation method to nonlinear heat equations arising in heat transfer, Phys. Lett. A 355, 337-341 (2006)

18. Ganji, D.D.; Jamshidi, N.: Application of energy balance method and variational iteration method to an oscillation of a mass attached to a stretched elastic wire, Int. J. N. Sc. N. Sim. 7, 413-420 (2006)

19. Sweilam, N.H.; Khader, M.M.: Variational iteration method for one dimensional nonlinear thermoelasticity, Chaos Soliton Fract. 32, 145-149 (2007)

20. He, J.H.: Variational iteration method-a kind of non-linear analytical technique: Some examples. Int. J. Non-Linear Mech. 34(4), 699-708 (1999)

21. Odibat, Z.M.; Momani, S.: Application of variational iteration method to nonlinear differential equations of fractional order. Int. J. Nonlinear Sci. 7, 27-34 (2006)

22. He, J.H.: Variational iteration method for autonomous ordinary differential systems, A Math. Com. 114, 115-123 (2000)

23. Ganji, D.D.; Jannatabadi, M.; Mohseni, E.: Application of Hes variational iteration method to nonlinear Jaulent-Miodek equations and comparing it with ADM, J. Comput. Appl. Math, 207(1), 35-45 (2006)

24. Abbasbandy, S.: Application of He's homotopy perturbation method to functional integral equations. Chaos, Solitons Fract. 31, 1243-1247 (2007)
25. Pakdemirli, M.; Karahan, M.M.F.; Boyaci, H.: A new perturbation algorithm with better convergence properties: multiple scales lindstedt Poincare method, J. Comput. Appl. Math. 14, 31-44 (2009)

26. Pakdemirli, M.; Karahan, M.M.F.: A new perturbation solution for systems with strong quadratic and qubic nonlinearities, Math. Meth. Appl. Sci. 33, 704-712 (2010)

27. Pakdemirli, M.; Karahan, M.M.F.; Boyaci, H.: Forced vibrations of strongly nonlinear systems with multiple scales lindstedt Poincare method, J. Appl. Math. Comput. 16(4), 879-889 (2011)

28. Pakdemirli, M.; Aksoy, Y.; Boyaci, H.: A new perturbation iteration approach for first order differential equations, J. Appl. Math. Comput. 16(4), 890-899 (2011)

29. Pakdemirli, M.; Sahin, A.Z.: Group classification of fin equation with variable thermal properties, Int. J. Eng. Sci. 42, 1875-1889 (2004)

30. Pakdemirli, M.; Sahin, A.Z.: Similarity analysis of a nonlinear fin equation, Appl. Math. Lett. 19, 378-384 (2006)

31. Kocak, H.; Yildirim, A.; Zhang, D.H.; Mohyud-Din, S.T.: The comparative boubaker polynomials expansion scheme (BPES) and Homotopy Perturbation Method (HPM) for solving astandard nonlinear second order boundary value problem, J. Comput. Math . model. 54, 417-422 (2011)

32. Raftari, B.; Yildirim, A.: Series solution of a nonlinear ODE Arising in Magnetohydrodynamic by HPM-Pade Technique, J. Comput. Appl. Math. 61, 1676-1681 (2011)

33. Khan, Y.; Faraz, N.; Yildirim, A.; Wu, Q.: A series of the long porous slider, Tribol. Trans. 54(2), 187-191 (2011) 